\title{
Imperative Remarks for "On Common Coupled Fixed Point Theorems for Comparable Mappings in Ordered Partially Metric Spaces" and an Answer to the Question: How to Smooth It Away
}

\author{
Hamed H. Alsulami, ${ }^{1}$ Erdal Karapınar, ${ }^{1,2}$ and Antonio Francisco Roldán López de Hierro ${ }^{3}$ \\ ${ }^{1}$ Nonlinear Analysis and Applied Mathematics Research Group (NAAM), King Abdulaziz University, Jeddah, Saudi Arabia \\ ${ }^{2}$ Department of Mathematics, Atilim University, Incek, 06836 Ankara, Turkey \\ ${ }^{3}$ University of Jaén, Campus las Lagunillas, s/n, 23071 Jaén, Spain
}

Correspondence should be addressed to Hamed H. Alsulami; hamed9@hotmail.com

Received 16 March 2014; Accepted 3 July 2014

Academic Editor: Sehie Park

Copyright (C) 2015 Hamed H. Alsulami et al. This is an open access article distributed under the Creative Commons Attribution License, which permits unrestricted use, distribution, and reproduction in any medium, provided the original work is properly cited.

We show that the main result in the work by Mutlu et al. is not true. We explain point by point some of its main mistakes and we propose an alternative version to smooth away the defects of it.

\section{Introduction}

Following Matthews [1], a partial metric on a nonempty set $X$ is a mapping $p: X \times X \rightarrow[0, \infty)$ verifying, for all $x, y, z \in X$,

$$
\begin{aligned}
& \left(\mathrm{P}_{1}\right) p(x, x) \leq p(x, y) ; \\
& \left(\mathrm{P}_{2}\right) p(x, x)=p(x, y)=p(y, y) \Longrightarrow x=y ; \\
& \left(\mathrm{P}_{3}\right) p(x, y)=p(y, x) ; \\
& \left(\mathrm{P}_{4}\right) p(x, z)+p(y, y) \leq p(x, y)+p(y, z) .
\end{aligned}
$$

In this case, $(X, p)$ is called a partial metric space. Although the authors of [2] used the notation $d$ for a partial metric space, we prefer using $p$ in order to avoid confusion with the metric case. Every metric space is a partial metric space, but the converse is false. For a partial metric $p$ on $X$, the mapping $d^{s}: X \times X \rightarrow[0, \infty)$, given by

$$
d^{s}(x, y)=2 p(x, y)-p(x, x)-p(y, y) \quad \forall x, y \in X,
$$

is a metric on $X$.

In [2], the authors introduced the following definition and announced the following theorem.
Definition 1 (Mutlu et al. [2], Definition 9). Assume that ( $X$, $\preccurlyeq)$ is a partially ordered set and $F, G: X \times X \rightarrow X$. F and $G$ mappings have the following properties:

if $n$ is even, then $F\left(x_{n}, y_{n}\right) \geqslant G\left(x_{n-1}, y_{n-1}\right)$ and

$$
F\left(y_{n}, x_{n}\right) \leqslant G\left(y_{n-1}, x_{n-1}\right) \text {; }
$$

if $n$ is odd, then $G\left(x_{n}, y_{n}\right) \geqslant F\left(x_{n-1}, y_{n-1}\right)$ and

$$
G\left(y_{n}, x_{n}\right) \leqslant F\left(y_{n-1}, x_{n-1}\right) \text {. }
$$

Theorem 2 (Mutlu et al. [2], Theorem 10). Suppose that ( $X$, $\preccurlyeq)$ is a partially ordered set and $p$ is a partial metric on $X$ with $(X, p)$ being a complete partial metric space. Assume that $F, G: X \times X \rightarrow X$ are satisfied by Definition 1 and also are continuous mappings possessing the mixed monotone property on $X$. Let there be a nonincreasing function $\varphi: \mathbb{R}^{+} \rightarrow \mathbb{R}$ such that $\varphi(t)<t$ and $\lim _{r \rightarrow t^{+}} \varphi(r)<t$ for all $t>0$ and also having $x \leqslant u$ and $y \geqslant v$, with

$$
\begin{aligned}
& p(F(x, y), G(u, v)) \\
& \quad \leq \varphi\left(\frac{p(x, u)+p(y, v)+p(x, v)+p(y, u)}{2}\right)
\end{aligned}
$$


for $x, y, u, v \in X$. If there exists $\left(x_{0}, y_{0}\right) \in X \times X$ with $x_{0} \preccurlyeq$ $F\left(x_{0}, y_{0}\right)$ and $y_{0} \geqslant F\left(y_{0}, x_{0}\right)$ at the time, $\exists x, y \in X$ with $x=$ $F(x, y)=G(x, y)$ and $y=F(y, x)=G(y, x)$.

In this paper, we show that Definition 1 is not clear. Therefore, Theorem 2 is not well posed. Furthermore, its proof has many mistakes. We illustrate that it fails with an example. Finally, we propose a correct version of Theorem 2.

\section{Preliminaries}

To better understand our main claims, let us introduce the following definitions and notation. In the sequel, $X$ will be a nonempty set and $X^{2}$ will represent the product space $X \times X$ of 2 identical copies of $X$.

Definition 3. A binary relation $\mathscr{R}$ on $X$ is a nonempty subset $\mathscr{R} \subseteq X \times X$. One will write $x \mathscr{R} y$ (or $x \leqslant y$ ) if $(x, y) \in \mathscr{R}$. A binary relation $\mathscr{R}$ on $X$ is reflexive if $x \mathscr{R} x$ for all $x \in X$, and it is transitive if $x \mathscr{R} z$ for all $x, y, z \in X$ such that $x \mathscr{R} y$ and $y \mathscr{R} z$. A reflexive and transitive relation on $X$ is a preorder (or a quasiorder) on $X$. If a preorder $\mathscr{R}$ is also antisymmetric ( $x \mathscr{R} y$ and $y \mathscr{R} x$ imply $x=y$ ), then $\mathscr{R}$ is called a partial order.

In [3], Guo and Lakshmikantham introduced the notion of coupled fixed point and, thus, they initiated the investigation of multidimensional fixed point theory.

Definition 4 (Guo and Lakshmikantham [3]). Let $F: X \times$ $X \rightarrow X$ be a given mapping. We say that $(x, y) \in X \times X$ is a coupled fixed point of $F$ if

$$
F(x, y)=x, \quad F(y, x)=y .
$$

Definition 5. Given two mappings $F, G: X \times X \rightarrow X$, we say that $(x, y) \in X \times X$ is a common coupled fixed point of $F$ and $G$ if

$$
F(x, y)=G(x, y)=x, \quad F(y, x)=G(y, x)=y .
$$

Henceforth, we will use the notation

$$
\begin{gathered}
\Phi=\{\varphi:[0, \infty) \longrightarrow[0, \infty): \varphi(t)<t, \\
\left.\lim _{r \rightarrow t^{+}} \varphi(r)<t \forall t>0\right\} .
\end{gathered}
$$

Functions in $\Phi$ are called comparison functions.

\section{Main Remarks about Theorem 2}

In the following lines, we must do the following commentaries in order to advise researchers about proving new results based on Theorem 2 .

(1) First of all, we point out that Definition 1 is not clear because it does not explain how the sequences $\left\{x_{n}\right\}$ and $\left\{y_{n}\right\}$ are. If they are arbitrary, then, for all $x, y, z, u \in X$,

$$
\begin{array}{ll}
F(x, y) \geqslant G(z, u), & F(y, x) \preccurlyeq G(u, z) ; \\
G(x, y) \geqslant F(z, u), & G(y, x) \preccurlyeq F(u, z) .
\end{array}
$$

Therefore, $F(x, y) \geqslant G(z, u) \geqslant F(x, y)$, so $F(x, y)=$ $G(z, u)$ for all $x, y, z, u \in X$. Hence, both mappings are constant, and the result is not interesting at all.

(2) As a consequence, Theorem 2 was incorrectly proved. Precisely, its proof collects very different mistakes.

(3) Although Theorem 2 assumes that $F$ and $G$ have the mixed monotone property, this condition was not used through its proof. We suppose that it is not necessary. Only Definition 1 is employed to prove that the iterative sequences $\left\{x_{n}\right\}$ and $\left\{y_{n}\right\}$ are monotone.

(4) The authors did not clarify if $\mathbb{R}^{+}$is either $[0, \infty)$ or $(0, \infty)$. In any case, the test function $\varphi: \mathbb{R}^{+} \rightarrow$ $\mathbb{R}$ can take arbitrary real values. It is clear that the contractivity condition (4) implies that $\varphi$ takes nonnegative values in different points, but it does not cover all possibilities. In particular, the function $\varphi$ is not declared at $t=0$. Then, $\varphi(0)$ can take any real value (its image is not restricted to $[0, \infty)$ ).

(5) The previous remark is important because if we take $x=y=u=v$ in (4), we deduce that

$$
p(F(x, x), G(x, x)) \leq \varphi(2 p(x, x)),
$$

which, in the metric case, let bound the distance $d(F(x, x), G(x, x))$ by $\varphi(0)$. If $\varphi(0)=0$, then $F(x, x)=$ $G(x, x)$ for all $x \in X$, which is a very strong restriction on the mappings $F$ and $G$.

(6) In [2], page 3, equation (15), the authors announced

$$
\begin{aligned}
p\left(x_{2 n+1}, x_{2 n+2}\right) & \\
=p( & \left.F\left(x_{2 n}, y_{2 n}\right), G\left(x_{2 n+1}, y_{2 n+1}\right)\right) \\
\leq \varphi & \left(\left(p\left(x_{2 n}, x_{2 n+1}\right)+p\left(y_{2 n}, y_{2 n+1}\right)\right.\right. \\
& \left.+p\left(x_{2 n}, y_{2 n+1}\right)+p\left(y_{2 n}, x_{2 n+1}\right)\right) \\
& \left.\times(2)^{-1}\right) \\
\leq \varphi & \left(\frac{p\left(x_{2 n}, x_{2 n+1}\right)+p\left(y_{2 n}, y_{2 n+1}\right)}{2}\right) .
\end{aligned}
$$

However, it is not clear why $p\left(x_{2 n}, y_{2 n+1}\right)=$ $p\left(y_{2 n}, x_{2 n+1}\right)=0$. Even if we would be able to prove that $x_{2 n}=y_{2 n+1}$ and $y_{2 n}=x_{2 n+1}$ (which was not proved), the condition $p(x, x)=0$ is not guaranteed in a partial metric space. Precisely, this is the characteristic property of partial metric spaces. Therefore, the second inequality in (10) is false.

(7) With respect to the previous remark, it is also necessary to point out that the contractivity condition (4) does not permit us to upper bound, for instance, the term $p\left(y_{2 n+1}, y_{2 n+2}\right)$. However, the authors affirmed in [2], page 3 , equation (16), that "Similarly, we can obtain

$p\left(y_{2 n+1}, y_{2 n+2}\right) \leq \varphi\left(\frac{p\left(x_{2 n}, x_{2 n+1}\right)+p\left(y_{2 n}, y_{2 n+1}\right)}{2}\right) . "$ 
Let us see where the mistake is. Theorem 2 only assumes that the inequality

$$
\begin{aligned}
& p(F(x, y), G(u, v)) \\
& \quad \leq \varphi\left(\frac{p(x, u)+p(y, v)+p(x, v)+p(y, u)}{2}\right)
\end{aligned}
$$

occurs provided that $x \leqslant u$ and $y \geqslant v$; that is, the first argument of $F$ must be $\preccurlyeq$-lower than the first argument of $G$. As the authors defined

$$
y_{2 n+1}=F\left(y_{2 n}, x_{2 n}\right), \quad y_{2 n+2}=G\left(y_{2 n+1}, x_{2 n+1}\right) \text {, }
$$

then

$p\left(y_{2 n+1}, y_{2 n+2}\right)=p\left(F\left(y_{2 n}, x_{2 n}\right), G\left(y_{2 n+1}, x_{2 n+1}\right)\right)$.

In this case, it was not proved that $y_{2 n} \leqslant y_{2 n+1}$ and $x_{2 n} \geqslant x_{2 n+1}$. In fact, the contrary inequalities were announced; that is, $y_{2 n} \geqslant y_{2 n+1}$ and $x_{2 n} \leqslant$ $x_{2 n+1}$. If both inequalities hold, then $\left(x_{2 n}, y_{2 n}\right)=$ $\left(x_{2 n+1}, y_{2 n+1}\right)$, which means that $\left(x_{2 n}, y_{2 n}\right)$ is a coupled fixed point of $F$. However, the proof must analyse the case in which $\left(x_{2 n}, y_{2 n}\right) \neq\left(x_{2 n+1}, y_{2 n+1}\right)$ for all $n \in \mathbb{N}$.

(8) Similarly, the contractivity condition (4) cannot be applied to study the term $p\left(x_{2 n}, x_{2 n+1}\right)$, because

$$
\begin{aligned}
p\left(x_{2 n}, x_{2 n+1}\right) & =p\left(G\left(x_{2 n-1}, y_{2 n-1}\right), F\left(x_{2 n}, y_{2 n}\right)\right) \\
& =p\left(F\left(x_{2 n}, y_{2 n}\right), G\left(x_{2 n-1}, y_{2 n-1}\right)\right)
\end{aligned}
$$

but, in this case, the inequalities $x_{2 n} \preccurlyeq x_{2 n-1}$ and $y_{2 n} \geqslant y_{2 n-1}$ cannot be proved in the case $\left(x_{2 n}, y_{2 n}\right) \neq$ $\left(x_{2 n-1}, y_{2 n-1}\right)$ since the contrary inequalities are supposed.

(9) When the authors tried to prove that the sequences $\left\{x_{n}\right\}$ and $\left\{y_{n}\right\}$ are Cauchy, as usual, they reasoned by contradiction. They announced that if $\left\{x_{n}\right\}$ is not Cauchy, then there exist $\varepsilon>0$ and two partial subsequences $\left\{x_{2 n(i)}\right\}$ and $\left\{x_{2 m(i)}\right\}$ such that

$$
2 n(i)>2 m(i)>i, \quad d\left(x_{2 m(i)}, x_{2 n(i)}\right) \geq \varepsilon,
$$

and if $2 n(i)$ is the smallest index verifying this property, then

$$
d\left(x_{2 m(i)}, x_{2 n(i)-1}\right)+d\left(y_{2 m(i)}, y_{2 n(i)-1}\right)<\varepsilon
$$

(see [2], page 3, equations (26) and (27)). However, the authors did not justify neither why we can suppose that the subindices are even nor why (17), involving the partial subsequences $\left\{y_{m(i)}\right\}$ and $\left\{y_{n(i)}\right\}$, can be deduced from (16), in which only $\left\{x_{m(i)}\right\}$ and $\left\{x_{n(i)}\right\}$ have a role. In [4], the authors justified the unidimensional case but did not study the coupled case.
(10) Other important mistakes can be found in [2], page 4, equation (39), where the author announced that

$$
\begin{aligned}
& d^{s}\left(x_{n}, x_{m}\right) \leq 2 p\left(x_{n}, x_{m}\right)=0, \\
& d^{s}\left(y_{n}, y_{m}\right) \leq 2 p\left(y_{n}, y_{m}\right)=0 .
\end{aligned}
$$

Taking into account that $d^{s}$ is a metric on $X$, if this property was true, then the sequences $\left\{x_{n}\right\}$ and $\left\{y_{n}\right\}$ would be constant for all $n \geq n_{0}$ which, in general, is false. In fact, it is well known that if there is some $n_{0} \in$ $\mathbb{N}$ such that $\left(x_{n_{0}+1}, y_{n_{0}+1}\right)=\left(x_{n_{0}}, y_{n_{0}}\right)$, then $\left(x_{n_{0}}, y_{n_{0}}\right)$ is the common coupled fixed point.

(11) Finally, we point out that Theorem 7 in [2] is incorrectly enunciated.

\section{An Example}

It is not clear how we can show a counterexample of Theorem 2 because Definition 1 is not well posed. Item 1 of Section 3 shows that, in general, it is a very restrictive hypothesis ( $F$ and $G$ must be constant and equal). Therefore, we are going to show an example in which other hypotheses hold, where $F$ and $G$ are not constant, but $F$ and $G$ have no common coupled fixed point.

Let $X=[0.9,2]$ provided with its usual partial order $\leq$ and let $p(x, y)=\max (x, y)$ for all $x, y \in X$. Then, $(X, p)$ is a complete partial metric space. Let us define $F, G: X^{2} \rightarrow X$ and $\varphi:[0, \infty) \rightarrow[0, \infty)$ by

$$
\begin{gathered}
F(x, y)=1+0.001 x, \quad G(x, y)=1+0.002 x \quad \forall x, y \in X, \\
\varphi(t)=0.99 t \quad \forall t \geq 0 .
\end{gathered}
$$

Then, $F$ and $G$ have the mixed monotone property, both mappings are continuous, and $\varphi \in \Phi$. Letting $x_{0}=0.9$ and $y_{0}=2$, we have the fact that $x_{0}=0.9 \leq 1.0009=F\left(x_{0}, y_{0}\right)$ and $y_{0}=2 \geq 1.004=F\left(y_{0}, x_{0}\right)$. However, the condition $F(x, y)=G(x, y)$ is impossible when $(x, y) \in X^{2}$, so $F$ and $G$ cannot have a common coupled fixed point. It only remains to prove that the contractivity condition (4) holds.

Let $x, y, u, v \in X$ be such that $x \leq u$ and $y \geq v$. As $x \leq u$, then $0.001 x \leq 0.002 u$, so

$$
\begin{aligned}
& p(F(x, y), G(u, v)) \\
& \quad=\max (1+0.001 x, 1+0.002 u)=1+0.002 u .
\end{aligned}
$$

On the other hand,

$$
\begin{gathered}
\varphi\left(\frac{p(x, u)+p(y, v)+p(x, v)+p(y, u)}{2}\right) \\
=\frac{0.99}{2}(\max (x, u)+\max (y, v) \\
\quad+\max (x, v)+\max (y, u)) \\
=0.495(u+y+\max (x, v)+\max (y, u)) .
\end{gathered}
$$


Therefore,

$$
\begin{aligned}
& p(F(x, y), G(u, v)) \\
& \quad \leq \varphi\left(\frac{p(x, u)+p(y, v)+p(x, v)+p(y, u)}{2}\right) \\
& \Longleftrightarrow 1+0.002 u \leq 0.495(u+y+\max (x, v)+\max (y, u)) \\
& \Longleftrightarrow 1 \leq 0.493 u+0.495(y+\max (x, v)+\max (y, u)) .
\end{aligned}
$$

Taking into account that

$$
\begin{aligned}
& 0.495(y+\max (x, v)+\max (y, u)) \\
& \geq 0.495(0.9+0.9+0.9)=1.3365,
\end{aligned}
$$

we conclude that inequality (22) holds.

\section{A Correct Version}

Taking into account the commentaries given in Section 3, we propose a correct version of Theorem 2. Item 6 shows that the terms $p(x, v)+p(y, u)$ must not be employed in the contractivity condition, and items 7-8 suggest that it is very difficult to use two different mappings $F$ and $G$ in the contractivity condition as we cannot compare, at the same time, the terms $p(F(x, y), G(u, v)), p(F(x, y), F(u, v))$, and $p(G(x, y), G(u, v))$. If $F$ and $G$ are not involved in the second member of the contractivity condition, it is almost impossible to control the term $p\left(x_{n}, x_{m}\right)+p\left(y_{n}, y_{m}\right)$ when $n$ and $m$ can be even and odd.

In recent times, many coupled/tripled/quadrupled/multidimensional fixed point theorems in various abstract metric spaces have come to be simple consequences of their corresponding unidimensional results (see, e.g., [5-10] and the references therein). Following this line of research, we present here a correct version of Theorem 2 for three reasons mainly: (1) for the sake of completeness; (2) to describe how coupled results in partial metric spaces can be deduced from the unidimensional case; (3) to show some possible hypotheses to ensure the existence of common coupled fixed points when we work with two different mappings. Before doing it, we need to introduce the following preliminaries.

Definition 6. Let $\preccurlyeq$ be a binary relation on $X$.

(i) Two points $x, y \in X$ are called $\preccurlyeq$-comparable if $x \leqslant y$ or $y \leqslant x$.

(ii) A subset $A \subseteq X$ is said to be $\preccurlyeq$-well ordered if every two points of $A$ are $\leqslant$-comparable.

(iii) A mapping $T: X \rightarrow X$ is called $\preccurlyeq$-nondecreasing if $x \leqslant y$ implies $T x \leqslant T y$.

Definition 7 . One will say that $(X, p, \preccurlyeq)$ is a partially ordered partial metric space (sometimes, it is also known as ordered partial metric space) if $p$ is a partial metric on $X$ and $\preccurlyeq$ is a partial order on $X$.
Definition 8 (Nashine et al. [4]). Let $(X, \preccurlyeq)$ be a partially ordered set. A pair of mappings $S, T: X \rightarrow X$ is said to be weakly increasing if $S x \leqslant T S x$ and $T x \leqslant S T x$ for all $x \in X$. The mapping $S$ is said to be $T$-weakly isotone increasing if for all $x \in X$, we have $S x \leqslant T S x \leqslant S T S x$.

Very recently, Nashine et al. [4] proved the following result.

Theorem 9 (Nashine et al. [4], Theorem 3.6). Let $(X, p, \preccurlyeq)$ be a complete partially ordered partial metric space. Let T, $S$ : $X \rightarrow X$ be two mappings such that

$$
p(T x, S y) \leq M(x, y)
$$

for all $\preccurlyeq$-comparable $x, y \in X$, where

$$
\begin{gathered}
M(x, y)=\max (\varphi(p(x, y)), \varphi(p(x, T x)), \varphi(p(y, S y)), \\
\left.\varphi\left(\frac{p(y, T x)+p(x, S y)}{2}\right)\right)
\end{gathered}
$$

and $\phi:[0, \infty) \rightarrow[0, \infty)$ is a continuous function with $\phi(t)<$ $t$ for each $t>0, \phi(0)=0$. We suppose the following:

(i) $S$ is T-weakly isotone increasing,

(ii) $S$ and $T$ are continuous.

Then, the set $\mathscr{F}(T, S)$ of common fixed points of $T$ and $S$ is nonempty, and $p(z, z)=p(T z, T z)=p(S z, S z)=p(z, S z)=$ $p(z, T z)=0$ for $z \in \mathscr{F}(T, S)$. Moreover, the set $\mathscr{F}(T, S)$ is well ordered if and only if $T$ and $S$ have one, and only one, common fixed point.

Based on this result, we present a coupled version that can be interpreted as a correct version of Mutlu et al's theorem.

Theorem 10. Let $(X, p, \preccurlyeq)$ be a complete partially ordered partial metric space and let $F, G: X^{2} \rightarrow X$ be two continuous mappings such that, for all $x, y, u, v \in X$ verifying $x \leqslant u, y \geqslant v$ or $x \geqslant u, y \leqslant v$,

$$
\begin{aligned}
& p(F(x, y), G(u, v)) \\
& \quad+p(F(y, x), G(v, u)) \leq M_{F, G}(x, y, u, v),
\end{aligned}
$$

where

$$
\begin{aligned}
M_{F, G}(x, y, u, v) & \\
=\max & \{\varphi(p(x, u)+p(y, v)), \\
& \varphi(p(x, F(x, y))+p(y, F(y, x))), \\
& \varphi(p(u, G(u, v))+p(v, F(v, u))), \\
& \varphi((p(x, G(u, v))+p(y, G(v, u))+p(u, F(x, y)) \\
& \left.+p(v, F(y, x)))) \times(2)^{-1}\right\} .
\end{aligned}
$$


And $\varphi:[0, \infty) \rightarrow[0, \infty)$ is a continuous function with $\phi(0)=0$ and $\phi(t)<t$ for each $t>0$. Also assume that for all $x, y \in X$, we have the fact that

$$
\begin{aligned}
G(x, y) & \leqslant F(G(x, y), G(y, x)) \\
& \preccurlyeq G(F(G(x, y), G(y, x)), F(G(y, x), G(x, y))), \\
G(y, x) & \geqslant F(G(y, x), G(x, y)) \\
& \geqslant G(F(G(y, x), G(x, y)), F(G(x, y), G(y, x))) .
\end{aligned}
$$

Then, the set $\mathscr{F}(F, G)$ of common coupled fixed points of $F$ and $G$ is nonempty, and

$$
\begin{aligned}
p(z, z) & =p(\omega, \omega)=p(z, F(z, \omega)) \\
& =p(\omega, F(\omega, z))=p(z, G(z, \omega)) \\
& =p(\omega, G(\omega, z))=p(F(z, \omega), F(z, \omega)) \\
& =p(F(\omega, z), F(\omega, z))=p(G(z, \omega), G(z, \omega)) \\
& =p(G(\omega, z), G(\omega, z))=0
\end{aligned}
$$

for all $(z, \omega) \in \mathscr{F}(F, G)$.

To prove it, we use the following notation and basic facts. Let $p$ be a partial metric on $X$ and define $p_{2}: X^{2} \times X^{2} \rightarrow$ $[0, \infty)$ by

$$
\begin{array}{r}
p_{2}((x, y),(u, v))=p(x, u)+p(y, v) \\
\forall(x, y),(u, v) \in X^{2} .
\end{array}
$$

Then, $\left(X^{2}, p_{2}\right)$ is a partial metric space. Now, let $\preccurlyeq$ be a binary relation on $X$ and define the relation $\sqsubseteq$ on $X^{2}$ by

$$
(x, y) \sqsubseteq(u, v) \Longleftrightarrow[x \leqslant u, y \geqslant v] .
$$

Then, $\sqsubseteq$ is also a binary relation on $X^{2}$ with the following property: if $\preccurlyeq$ is a partial order on $X$, then $\sqsubseteq$ is a partial order on $X^{2}$.

Given two mappings $F, G: X^{2} \rightarrow X$, let us denote by $T_{F}^{2}, T_{G}^{2}: X^{2} \rightarrow X^{2}$ the mappings

$$
\begin{array}{r}
T_{F}^{2}(x, y)=(F(x, y), F(y, x)), \\
T_{G}^{2}(x, y)=(G(x, y), G(y, x)) \\
\forall(x, y) \in X^{2} .
\end{array}
$$

If $F$ is $p$-continuous, then $T_{F}^{2}$ is $p_{2}$-continuous. Using the notation given in (25), the contractivity condition (26) can be rewritten as (24) in the sense that

$$
p_{2}\left(T_{F}^{2}(x, y), T_{G}^{2}(u, v)\right) \leq M((x, y),(u, v))
$$

for all $(x, y),(u, v) \in X^{2}$ such that $(x, y) \sqsubseteq(u, v)$ or $(u$, $v) \sqsubseteq(x, y)$ (i.e., $\sqsubseteq$-comparable points of $\left.X^{2}\right)$. Furthermore, inequalities (28) are equivalent to

$$
T_{G}^{2}(x, y) \sqsubseteq T_{F}^{2} T_{G}^{2}(x, y) \sqsubseteq T_{G}^{2} T_{F}^{2} T_{G}^{2}(x, y) \quad \forall(x, y) \in X^{2} ;
$$

that is, $T_{G}^{2}$ is $T_{F}^{2}$-weakly isotone increasing in the partially ordered set $\left(X^{2}\right.$, ㄷ). Applying Theorem 9, the set $\mathscr{F}\left(T_{F}^{2}, T_{G}^{2}\right)$ of common fixed points of $T_{F}^{2}$ and $T_{G}^{2}$ is nonempty, and $p_{2}((z, \omega),(z, \omega))=p_{2}\left(T_{F}^{2}(z, \omega), T_{F}^{2}(z, \omega)\right)=p_{2}\left(T_{G}^{2}(z, \omega)\right.$, $\left.T_{G}^{2}(z, \omega)\right)=p_{2}\left((z, \omega), T_{F}^{2}(z, \omega)\right)=p_{2}\left((z, \omega), T_{G}^{2}(z, \omega)\right)=0$ for $(z, \omega) \in \mathscr{F}\left(T_{F}^{2}, T_{G}^{2}\right)$. Notice that a common fixed point of $T_{F}^{2}$ and $T_{G}^{2}$ is nothing but a common coupled fixed point of $F$ and $G$. This means that the set $\mathscr{F}(F, G)$ of common coupled fixed points of $F$ and $G$ is nonempty and

$$
\begin{aligned}
p(z, z) & =p(\omega, \omega)=p(z, F(z, \omega)) \\
& =p(\omega, F(\omega, z))=p(z, G(z, \omega))=p(\omega, G(\omega, z)) \\
& =p(F(z, \omega), F(z, \omega))=p(F(\omega, z), F(\omega, z)) \\
& =p(G(z, \omega), G(z, \omega))=p(G(\omega, z), G(\omega, z))=0
\end{aligned}
$$

for all common coupled fixed points $(z, \omega) \in X^{2}$ of $F$ and $G$.

\section{Conclusions}

We first note that we can suggest further corrected forms for the paper [2]. We prefer Theorem 10 since it is the best possible corrected result inspired from the very defective main result in [2], that is, Theorem 2.

Secondly, we can list several consequences of Theorem 10, for instance, by taking $F=G$ and/or by replacing $\varphi(t)=k t$ with $0 \leq k<1$. One can also get several corollaries by replacing $M_{F, G}(x, y, u, v)$ with the various combinations of the terms in $M_{F, G}(x, y, u, v)$. Furthermore, it is easy to state the analog of Theorem 10 in the context of "complete partial metric space," instead of "complete partially ordered partial metric space." Regarding the skeleton of the paper, we avoid listing all these results that can be easily derived by the reader.

Finally, independently from the structure of the abstract space (e.g., metric space, partial metric space, G-metric space, $b$-metric space, etc.), we underline the fact that multidimensional fixed point theorems and, in particular, coupled fixed point theorems can be derived from the existing corresponding results in the literature (see, e.g., [5-8]).

\section{Conflict of Interests}

The authors declare that they have no competing interests.

\section{Authors' Contribution}

All authors contributed equally and significantly in writing this paper. All authors read and approved the final paper.

\section{Acknowledgments}

This research was supported by the Deanship of Scientific Research (DSR), King Abdulaziz University, Jeddah, Saudi Arabia. The authors thank the anonymous referees for their remarkable comments, suggestions, and ideas that helped to improve this paper. Antonio Francisco Roldán López de 
Hierro has been partially supported by Junta de Andalucía by project FQM-268 of the Andalusian CICYE.

\section{References}

[1] S. G. Matthews, "Partial metric topology, general topology and its applications," in Proceedings of the 8th Summer Conference of Queen's College, vol. 728, pp. 183-197, Annals of the New York Academy of Sciences, 1994.

[2] A. Mutlu, N. Yolcu, B. Mutlu, and N. Bildik, "On common coupled fixed point theorems for comparable mappings in ordered partially metric spaces," Abstract and Applied Analysis, vol. 2014, Article ID 486384, 6 pages, 2014.

[3] D. J. Guo and V. Lakshmikantham, "Coupled fixed points of nonlinear operators with applications," Nonlinear Analysis: Theory, Methods \& Applications, vol. 11, no. 5, pp. 623-632, 1987.

[4] H. K. Nashine, Z. Kadelburg, and S. Radenovi c', "Common fixed point theorems for weakly isotone increasing mappings in ordered partial metric spaces," Mathematical and Computer Modelling, vol. 57, no. 9-10, pp. 2355-2365, 2013.

[5] R. Agarwal, E. Karapınar, and A. Roldán, "Fixed point theorems in quasi-metric spaces and applications to coupled/tripled fixed points on $G^{*}$-metric spaces," Journal of Nonlinear and Convex Analysis. In press.

[6] A. Roldán, J. Martínez-Moreno, C. Roldán, and E. Karapınar, "Some remarks on multidimensional fixed point theorems," Fixed Point Theory, vol. 15, pp. 545-558, 2014.

[7] B. Samet, E. Karapınar, H. Aydi, and V. Ć. Rajić, "Discussion on some coupled fixed point theorems," Fixed Point Theory and Applications, vol. 2013, article 50, 2013.

[8] R. P. Agarwal and E. Karapınar, "Remarks on some coupled fixed point theorems in G-metric spaces," Fixed Point Theory and Applications, vol. 2013, no. 2, 2013.

[9] S. Radenović, "Remarks on some recent coupled coincidence point results in symmetric G-metric spaces," Journal of Operators, vol. 2013, Article ID 290525, 8 pages, 2013.

[10] M. Jleli and B. Samet, "Remarks on G-metric spaces and fixed point theorems," Fixed Point Theory and Applications, vol. 2012, article 210, 2012. 


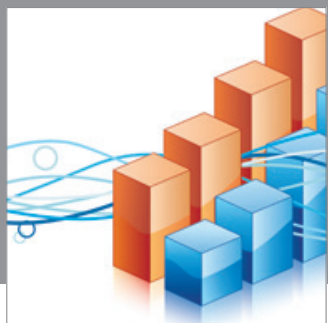

Advances in

Operations Research

mansans

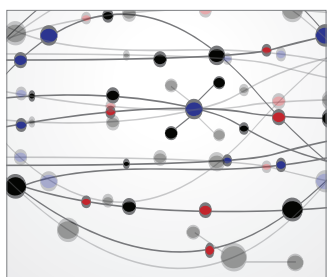

The Scientific World Journal
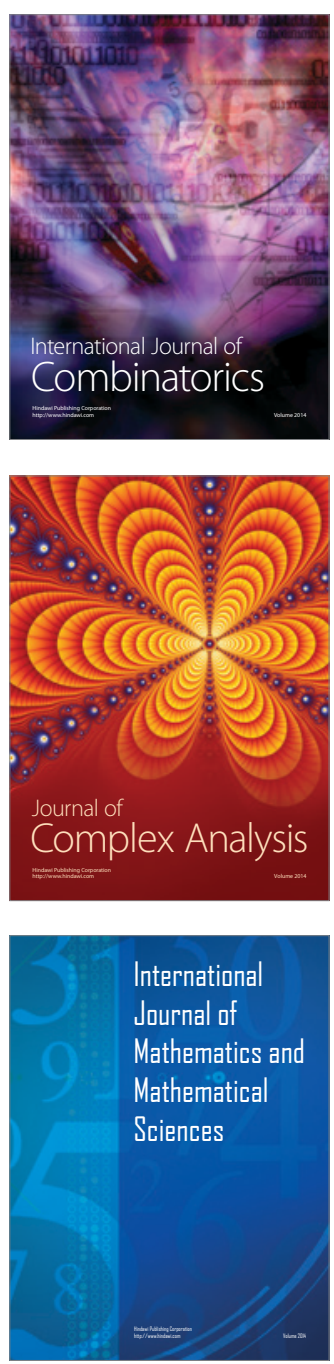
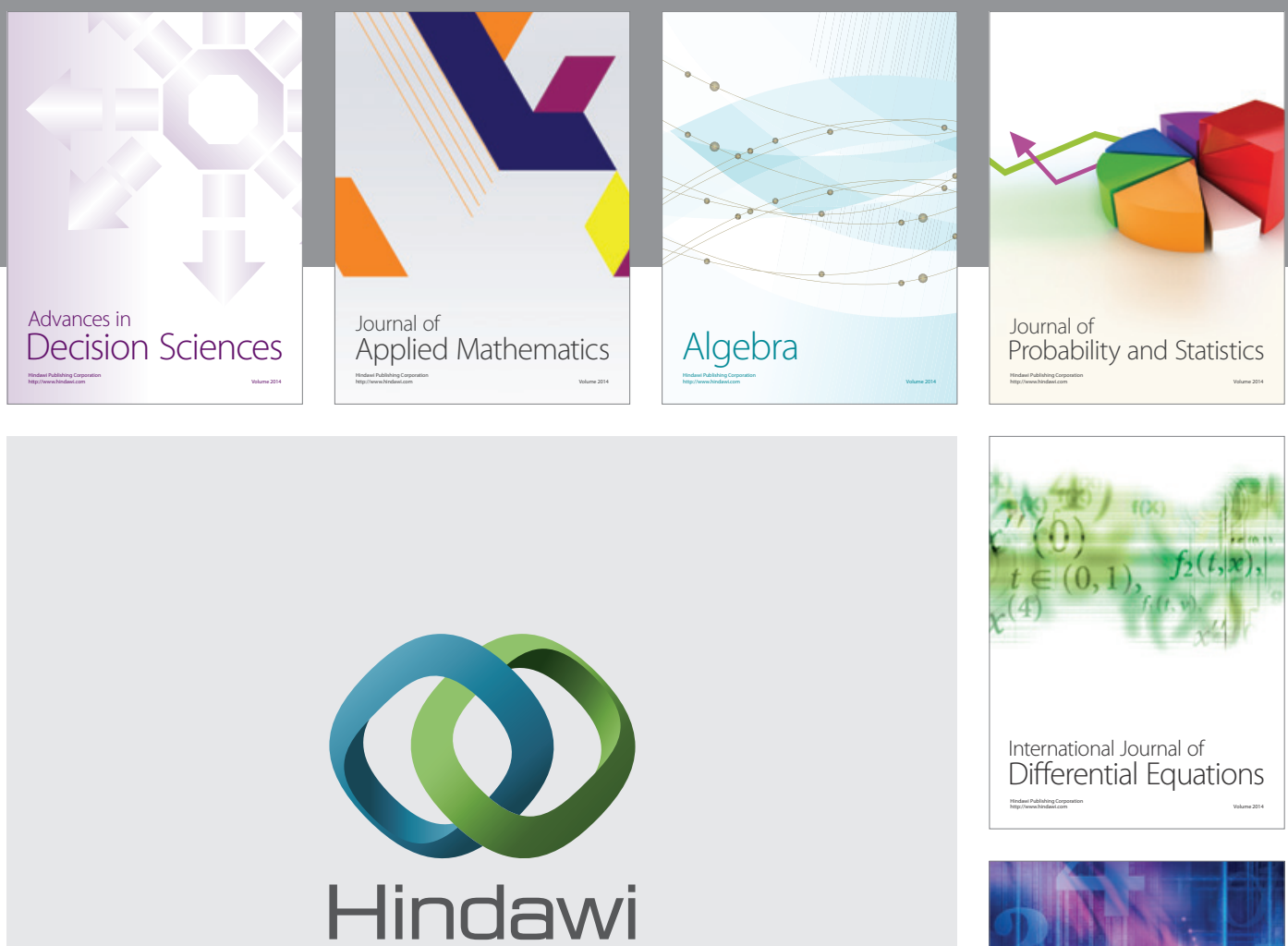

Submit your manuscripts at http://www.hindawi.com
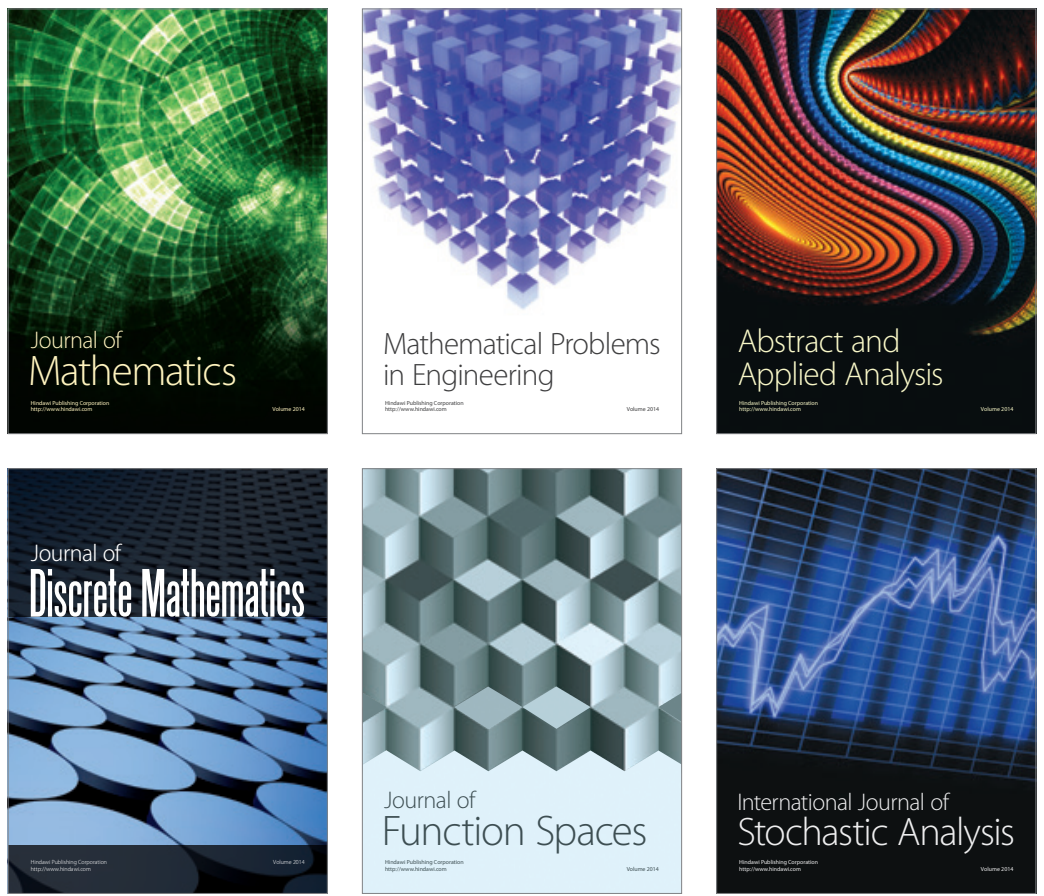

Journal of

Function Spaces

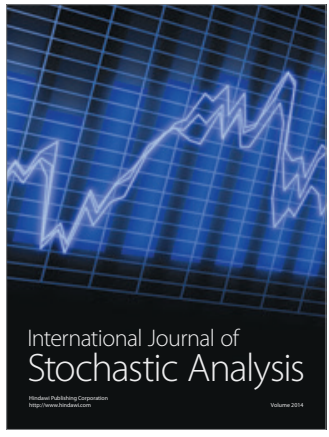

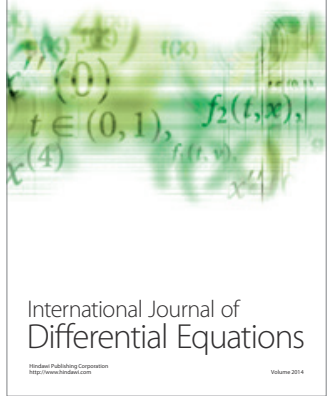
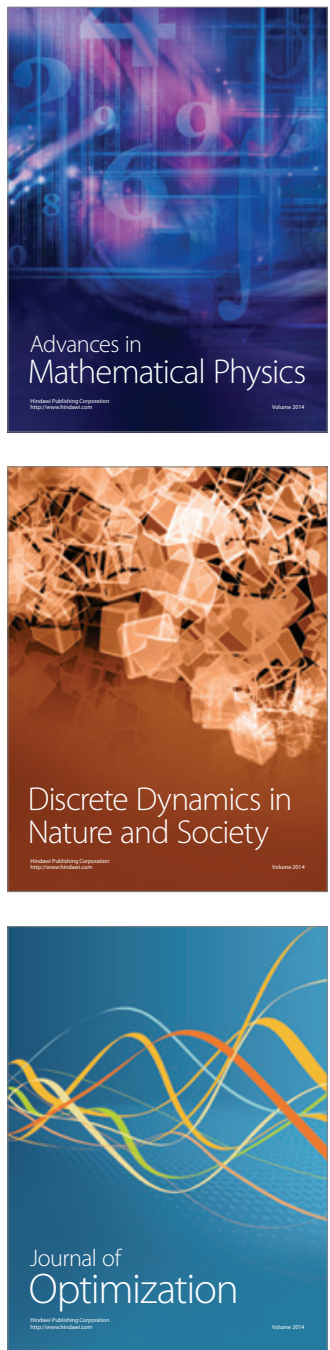